\title{
Crizotinib inhibits NF2-associated schwannoma through inhibition of focal adhesion kinase 1
}

\author{
Scott Troutman ${ }^{1}$, Susana Moleirinho ${ }^{1}$, Smitha Kota ${ }^{1}$, Kendall Nettles ${ }^{1}$, Mohammad \\ Fallahi $^{2}$, Gary L. Johnson ${ }^{3}$, Joseph L. Kissil ${ }^{1}$ \\ ${ }^{1}$ Department of Cancer Biology, The Scripps Institute, Jupiter, FL, 33458, USA \\ ${ }^{2}$ Department of Informatics Core, The Scripps Institute, Jupiter, FL, 33458, USA \\ ${ }^{3}$ Department of Pharmacology, University of North Carolina, Chapel Hill, NC, 27599, USA
}

Correspondence to: Joseph L. Kissil, email: jkissil@scripps.edu

Keywords: neurofibromatosis, NF2, signal transduction, crizotinib, FAK

Received: January 04, 2016

Accepted: May 13, 2016

Published: June 23, 2016

\section{ABSTRACT}

Neurofibromatosis type 2 (NF2) is a dominantly inherited autosomal disease characterized by schwannomas of the 8th cranial nerve. The NF2 tumor suppressor gene encodes for Merlin, a protein implicated as a suppressor of multiple cellular signaling pathways. To identify potential drug targets in NF2-associated malignancies we assessed the consequences of inhibiting the tyrosine kinase receptor MET. We identified crizotinib, a MET and ALK inhibitor, as a potent inhibitor of NF2-null Schwann cell proliferation in vitro and tumor growth in vivo. To identify the target/s of crizotnib we employed activity-based protein profiling (ABPP), leading to identification of FAK1 (PTK2) as the relevant target of crizotinib inhibition in NF2-null schwannoma cells. Subsequent studies confirm that inhibition of FAK1 is sufficient to suppress tumorigenesis in animal models of NF2 and that crizotinib-resistant forms of FAK1 can rescue the effects of treatment. These studies identify a FDA approved drug as a potential treatment for NF2 and delineate the mechanism of action in NF2-null Schwann cells.

\section{INTRODUCTION}

Neurofibromatosis type 2 (NF2) is a dominantly inherited autosomal disease (affecting 1 in 30,000), attributed to the loss-of-heterozygosity ( $\mathrm{LOH}$ ) of the $N F 2$ gene. The disease is characterized mainly by development of Schwann cell tumors of the eighth cranial nerve. Mutations and loss of heterozygosity ( $\mathrm{LOH}$ ) of the $N F 2$ locus have also been detected at high frequency in various tumors of the nervous system, including sporadic schwannomas, meningiomas and ependymomas [1]. The NF2 tumor suppressor gene encodes a $69-\mathrm{kDa}$ protein called Merlin (Moesin, ezrin, and radixin like protein), implicated in the regulation of a number of the Rac1, Ras/MAPK, mTOR and Hippo signaling pathways [2]. Previous studies have demonstrated that Merlin interacts with CD44 and inhibits signaling through the MET proto-oncogene, a receptor tyrosine kinase [3]. In addition, analysis of $N F 2$-null vestibular schwannomas demonstrated dysregulation of MET expression and other associated genes [4]. Moreover, several studies have demonstrated that Merlin functions as an inhibitor of the group I p21-activated kinases (PAKs), by direct inhibition and by inhibition of the small G-protein Rac1, a direct upstream activator of the PAKs [5-8]. Previous studies have shown that the p21-activated kinase 1 (PAK1) activates MET signaling and that disruption of this activity inhibits PAK1-driven anchorage-independent cell growth [9]. This is especially significant in the case of NF2, as previous work has established the PAKs as necessary effectors and targets for inhibition in NF2 $[6,10]$. These findings implicate MET as a potential target for therapeutic inhibition in the context of NF2 loss. We therefore set out to determine whether MET inhibition would be beneficial in a model of NF2-associated schwannoma.

Towards this goal we employed pharmacologic and genetic approaches to inhibit MET and identified the FDA approved drug crizotinib (PF-2341066), as a potent inhibitor of tumor progression and cellular proliferation of NF2-null Schwann cells. Moreover, our studies establish the inhibition of FAK1 (PTK2) as the primary mechanism 
of action for crizotinib in these cells. As crizotinib is well tolerated and is currently being evaluated in clinical trials in pediatric settings, our findings provide a strong rationale for assessing this drug in NF2 patients.

\section{RESULTS}

\section{Crizotinib inhibits schwannoma cell proliferation and tumor formation in vivo}

As previous studies implicated signaling downstream of MET as a potential therapeutic target in vestibular schwannoma [3, 4], we assessed the consequences of inhibiting MET in NF2-null Schwann cells. HEI193 cells (NF2-null, derived from a schwannoma of an NF2 patient), SC4 cells (Nf2-null mouse cells) and hSC2 $\lambda$ shNF2 cells (hSC2 $\lambda$ cells - immortalized normal human Schwann cells with stable knockdown of NF2) were treated with crizotinib (PF-2341066), a FDA approved drug that is a potent inhibitor of MET, ALK and Ros-1 [11]. Treatment of these cells resulted in a dose-dependent reduction in cell numbers over a period of 72 hours (Figure 1A-1C). To more accurately assess the inhibitory activity of crizotinib, we performed dose-response studies and established the $\mathrm{EC}_{50} \mathrm{~s}$ as $54 \mathrm{nM}$ and $230 \mathrm{nM}$ in SC4 or HEI193 cells, respectively (Figure 1D-1E). To determine if the effects of crizotinib are due to reduced cell proliferation or increased cell death rates, we assessed BrdU incorporation in treated SC4 cells. BrdU incorporation was significantly reduced over 72-hours, indicating that crizotinib has a strong effect on cellular proliferation (Figure 1F). To assess the potential effects of crizotinib on cell death, we examined the status of a number of apoptotic markers in the treated cells. We did not observe any differences in levels of cleaved Caspase-3, Caspase-7 and PARP between SC4 cells treated with crizotinib or vehicle only (not shown). These data suggest the effects of crizotinib are mediated primarily through inhibition of cell proliferation.

To assess if crizotinib can inhibit tumor growth in vivo, we employed an orthotopic model of NF2 that recapitulates the tumor microenvironment of schwannomas, by injection of luciferase-tagged NF2-null SC4 cells into a myelinated nerve $[12,13]$. In this model, tumors are first allowed to form and only then treatment is commenced. Beginning at 3 days post surgery, tumor progression was monitored every three days by bioluminescence imaging (BLI) and total flux counts were recorded for each animal. Ten days post injection, similar flux readings for all animals were validated and animals were then enrolled randomly into control (vehicle only) or drug-treated cohorts and were treated $(100 \mathrm{mg} / \mathrm{kg}$, IP, once daily) for a period of 24 days. Analysis of the flux reading for the animals in the cohorts indicated the trends of tumor growth are significantly different between the cohorts and that crizotinib treated mice displayed a significantly slower tumor growth rate (Figure 2A-2B). After 24 days of treatment the animals were sacrificed and the tumors removed and weighed. Comparison of the cohorts demonstrates a significantly lower average tumor weight in the crizotinib-treated group compared to control group (Figure 2C). Taken together, these data demonstrate that crizotinib has significant anti-proliferative activity against NF2-null Schwann cells in vitro and anti-tumor activity in vivo.

\section{MET is dispensable for NF2-null schwann cell proliferation}

To determine whether the effects of crizotinib are mediated through inhibition of MET, we employed pharmacological and genetic approaches to target MET more precisely. HEI193 or SC4 cells were treated with capmatinib (INC280), a potent MET inhibitor with reported average $\mathrm{IC}_{50}$ of $0.13 \mathrm{nmol} / \mathrm{L}$. INC280 is highly selective towards MET and at $2 \mu \mathrm{mol} / \mathrm{L}$ exhibited $30 \%$ or less inhibition against a panel of 57 structurally diverse kinases [14]. Treatment of SC4 or HEI193 cells with doses of up to $10 \mu \mathrm{M}$ and up to 72 hours had negligible effects on the proliferation of treated cells compared to controls (Supplementary Figure S1A-1B). To assess the requirement for MET directly, genome editing using the CRISPR/ Cas9 system was used to inactivate both MET alleles in SC4 cells, generating $\mathrm{SC}^{\mathrm{MET}(-)}$ cells (Supplementary Figure S1C). Inactivation of MET had a minimal effect on the proliferation of SC4 ${ }^{\mathrm{MET}(-)}$ cells compared to controls (Supplementary Figure S1D). Similar results were obtained using 2 independent siRNA oligos to knockdown the expression of MET (Not shown). Finally, treatment of SC4 ${ }^{\mathrm{MET}(-)}$ cells with crizotinib resulted in nearly identical activity compared to SC4 cells, suggesting the activity of crizotinib was not mediated through MET inhibition (Supplementary Figure S1D).

\section{Crizotinib inhibits a broad spectrum of kinases in $N F 2$-null schwannoma cells}

As the anti-proliferative effects of crizotinib on SC4 and HEI193 do not appear to be mediated through inhibition of MET, we applied a combination of unbiased approaches to identify the relevant targets. First, we examined the expression of kinases in SC4 cells through unbiased RNA sequencing (RNA-Seq) reads. The normalized read-counts of all annotated kinases revealed a broad pattern of expression of kinases from all families. Interestingly, the typical targets of crizotinib ALK and Ros-1 do not appear to be expressed in SC4 cells (Figure 3A and Supplementary Table S1). Western blot analysis of protein extracts from SC4 and HEI193 to assess the expression of ALK and Ros- 1 confirmed the lack of expression (not shown). To identify the kinases inhibited by crizotinib we employed activity- 
based protein profiling (ABPP). Briefly, we used an affinity column composed of multiple kinase inhibitors conjugated to Sepharose beads. This Multiplexed Inhibitor Bead (MIB) matrix provides the capability to capture $>85 \%$ of known protein kinases. The kinases were then eluted from the MIB matrix and identified and quantitated by mass spectrometry. This approach allowed us to compare multiple samples in a single run $[15,16]$. Comparison of the kinases captured by the MIB matrix to the RNA-seq expression data indicates the matrix captured the vast majority of kinases expressed (at least at the transcriptional level) in the SC4 cells (Figure 3B).

We next treated SC4 cells with crizotinib and collected cells at $0^{\prime}, 30^{\prime}, 240^{\prime}$ and 24 hours post treatment. Protein extracts from the different time points were analyzed by ABPP, as described above, and levels of captured kinases were assessed over time. The binding to crizotinib should prevent binding of an inhibited kinase to the MIB matrix and therefore levels of the kinase in treated fractions will be decreased (Supplementary Table S2). We applied the following criteria to identify targets of crizotinib that might mediate the effects observed in SC4 cells: First, target displays $>2$ fold reduction relative to untreated cells and, second, the reduction is sustained up to 24 hours. Applying these selection criteria resulted in the identification of 10 kinases: NUAK1, NDKM, PLK4, TESK1, ATR, SLK, PTK2, PTK2B, EPHA2 and STK10 (Figure 3C).

\section{Crizotinib's anti- proliferative activity is mediated by inhibition of FAK1/PTK2}

Although the ABPP identified at least ten potential candidates for crizotinib's activity, given the previous reports correlating NF2 deficiency to sensitivity of FAK1 inhibition $[17,18]$ we focused initially on FAK1 as the potential relevant crizotinib target in NF2-null schwannoma cells. To confirm that indeed crizotinib inhibits FAK1 in SC4 and HEI193 cells we treated the cells and assessed FAK1 activation by western blot analysis using a phospho-specific antibody against FAK-Y397, which is phosphorylated in activated FAK1. As expected, treatment with crizotinib resulted in decreased FAK-Y397 phosphorylation indicating it inhibits the activation of FAK1 in these cells (Figure 4A). To determine if FAK1 is required for proliferation of SC4 or HEI193 cells we employed shRNA or siRNA, respectively, to knockdown expression of FAK1 in these cells. Indeed, the knockdown of FAK1 significantly impaired the proliferation of these cells (Figure 4B-4C). In addition, we examined the effects of treating SC4 or HEI193 cells with small-molecule FAK1 inhibitors. Treatment of SC4 or HEI193 cells with defactinib (VS6303) or PND-1186 (VS-4718) significantly impaired cell proliferation in a dose-dependent manner (Figure 4D and not shown).

As the loss of function studies demonstrated crizotinib inhibits FAK1 in NF2-null Schwann cells

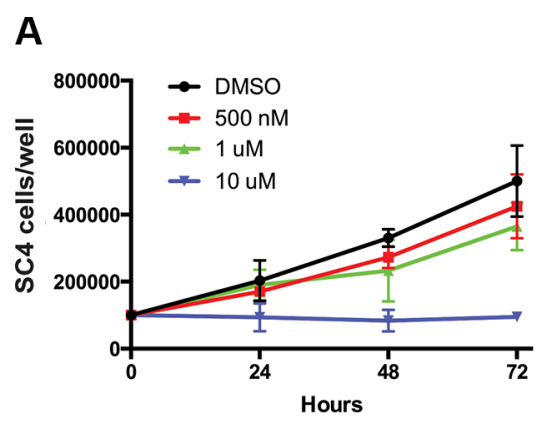

D

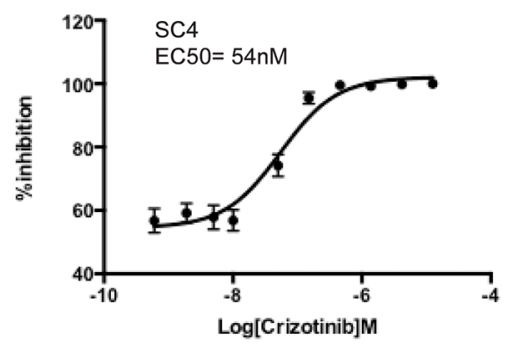

B

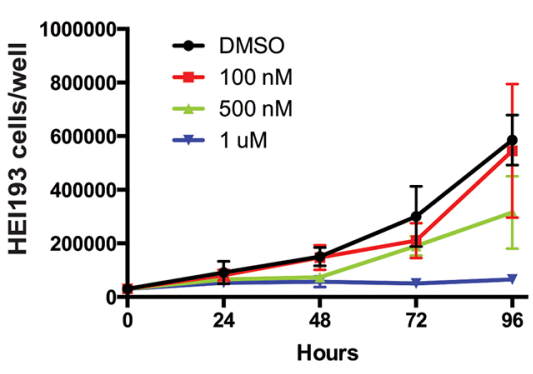

$\mathbf{E}$

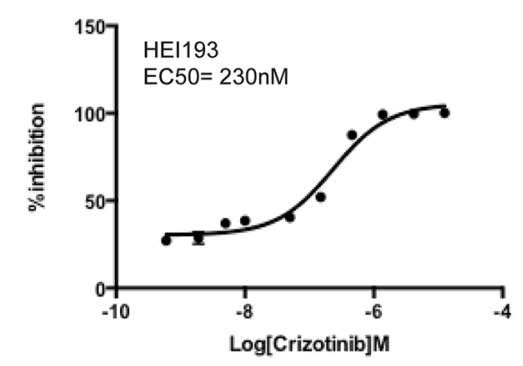

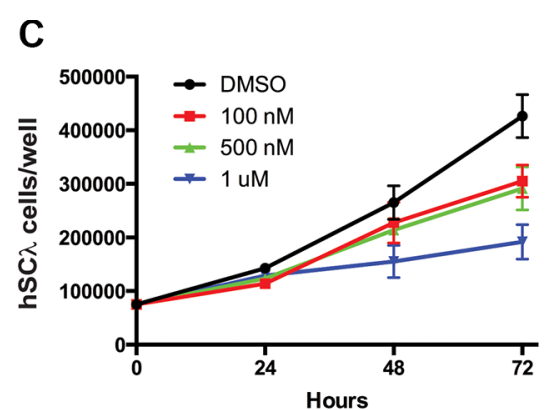

$\mathbf{F}$

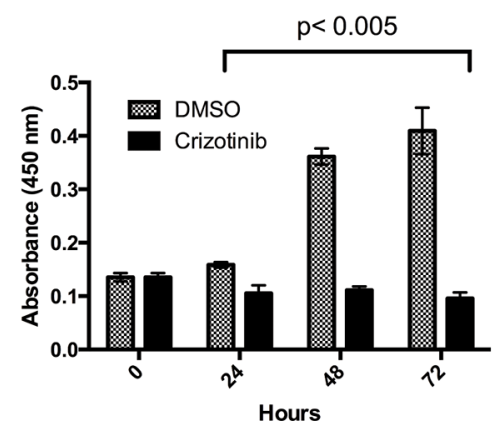

Figure 1: Impact of crizotinib treatment on NF2-null schwann cell proliferation. (A) SC4, (B) HEI193 or (C) hSC2 $\lambda$-shNF2 cells treated with crizotinib at the indicated concentrations or with $25 \%$ DMSO control, daily for 3 days. (D-E) 10-point dose response curves assessing viability of SC4 or HEI193 cells treated with Crizotinib at the indicated concentrations. (F) The proliferation of SC4 cells was assessed by BrdU incorporation at 24, 48 and 72 hours post crizotinib treatment $(1 \mu \mathrm{M})$ compared to control (DMSO). In all counting experiments cell numbers were scored daily and each time point was done in triplicate. The data shown is the mean of 3 independent experiments. Error bars $=\mathrm{SD}$. 
and is required for the proliferation of these cells, we sought to confirm these observations through gain of function approaches. Towards this aim, we first identified ALK mutations previously shown to confer a decreased sensitivity to crizotinib and assessed their conservation in FAK1. Mutations at residue S1206, located at the solvent front of the kinase domain, and at G1269, located in proximity to the DFG motif, have been suggested to diminish the affinity of ALK to crizotinib and facilitate cell survival in response to treatment [19, 20]. Both S1206 and G1269 are located in regions of ALK that are highly conserved in FAK1 with the equivalent residues at Serine 509 and Glycine 563, respectively (Figure 5A). We thus generated stable SC4 cells expressing FAK $1^{\text {wt }}$, FAK $1^{\text {S509R }}$, FAK $1^{\mathrm{G} 563 \mathrm{~S}}$ and FAK $1^{\text {S509R/G563S }}$ and assessed the expression levels and activity of the different FAK1 alleles. The expression levels of FAK1 are similar for the wild type and mutated alleles as are the levels of activation, as determined by phosphorylation of the Y397 autophosphorylation site (Figure 5B). To determine whether the mutated alleles confer resistance to crizotinib we assessed the $\mathrm{EC}_{50} \mathrm{~s}$ for $\mathrm{SC} 4$ cells stably expressing the different alleles. In a similar manner to what has been previously reported for ALK, the FAK1 S509R, G563S and S509R/G563S alleles conferred resistance to crizotinib treatment. While expression of wild type FAK1 increased the $\mathrm{EC}_{50}$ by 2-fold, the expression of the mutated FAK1 alleles shifted the $\mathrm{EC}_{50}$ by an order of magnitude (Figure 5C). Thus, the results from both loss- and gain-offunction experiments strongly implicate FAK1 inhibition as the main mechanism through which crizotinib mediates its effects in NF2-null schwannoma cells.

\section{DISCUSSION}

In spite of more than 2 decades of research into the molecular basis of NF2, treatment options are still lacking. Current treatment options are antisymptomatic, are limited to radiation or surgery and associated with severe morbidity. In an effort

A

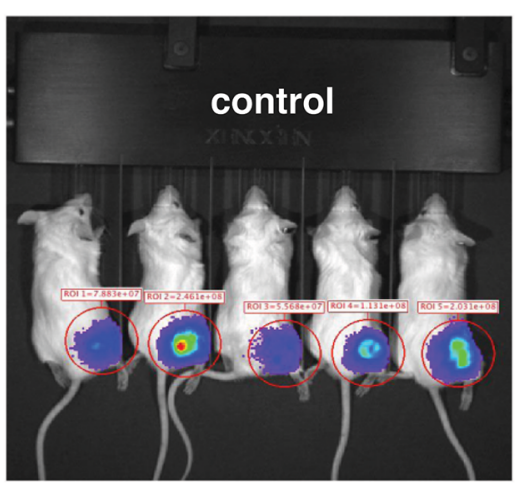

B

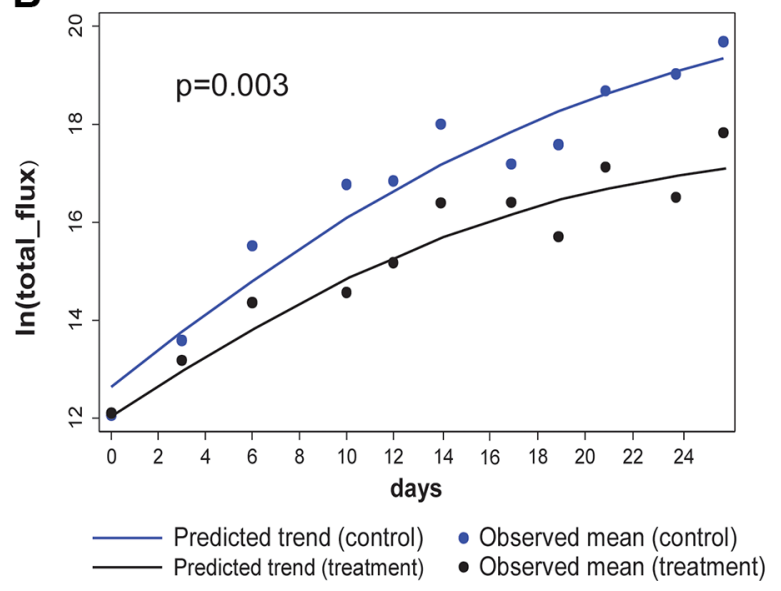

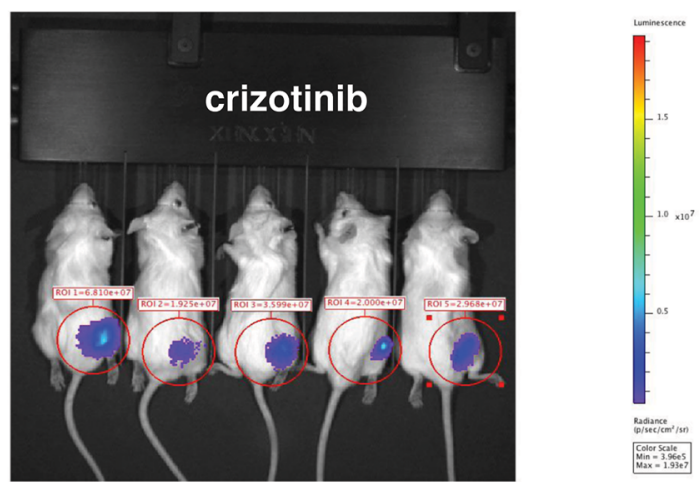

C

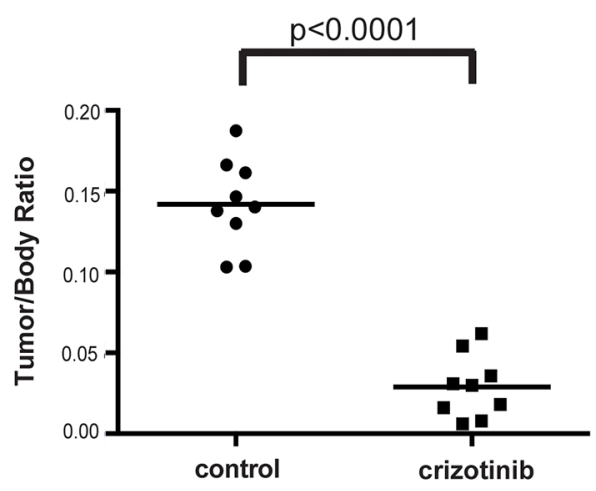

Figure 2: Crizotinib inhibits tumor growth in vivo. (A) Representative images from bioluminescence imaging (BLI) of mice carrying orthotopic tumors treated with crizotinib $(50 \mathrm{mg} / \mathrm{kg})$ or vehicle control $(25 \% \mathrm{DMSO})$ at day 14 of treatment. NOD/SCID mice were injected intraneurally with $5 \times 10^{4} \mathrm{SC} 4 / \mathrm{pLuc}$-mCherry cells and were enrolled into treatment after 10 days. Mice were treated daily for 25 days and imaged every 3 days to follow tumor development. (B) Quantitative analysis of the flux reading from treated cohorts. A mixed-effect model analysis indicated that the speed of tumor growth in treatment group is significantly slower than that in control group $(p=0.003)$. (C) Distribution of tumor/body weight ratio in the cohorts treated with crizotinib or vehicle control. The results of $t$-test with equal variances show that the crizotinib-treated group has significant lower average tumor weight than that observed in control group $(p<0.0001)$. For the in vivo experiments the $N=9$ in each cohort. 
to identify suitable therapeutic targets, we initially focused on the MET tyrosine kinase inhibitor, based on previous reports implicating it as a potential target $[3,4,9]$. While our findings indicate MET is mostly dispensable for the proliferation of NF2-null Schwann cells, our efforts identified crizotinib (Xalkori ${ }^{\circledR}$ - Pfizer) as a having significant anti-proliferative activity both in vitro and in vivo. Crizotinib was developed as an ATPcompetitive inhibitor of the anaplastic lymphoma kinase (ALK) and the MET/hepatocyte growth factor receptor (HGFR) [11, 21]. However, it has been shown to bind and inhibit multiple additional targets including the ROS1 and RON receptor tyrosine kinases [22-24].

As the primary targets of crizotinib inhibition do not appear to be expressed in NF2-null Schwann cells, we employed ABPP to identify 10 kinases that appear to be significantly inhibited by crizotinib. Although it is possible that any of these kinases, either alone or in combination, represents the target through which crizotinib mediates its anti-tumor effects, the recently reported association between NF2-status and sensitivity to FAK1 inhibition (see below) led us to focus on FAK1 as the potential target. Moreover, previous reports using

A

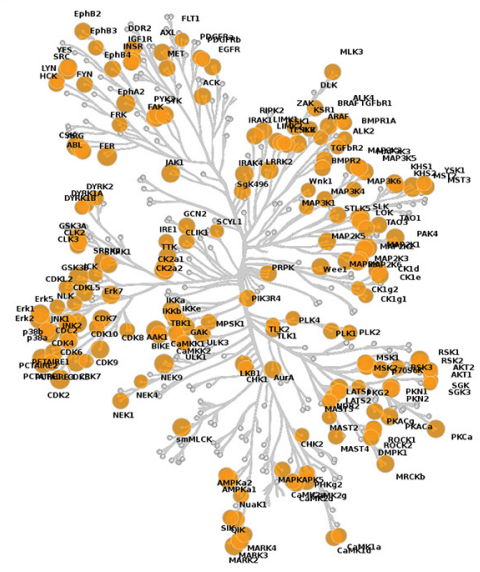

C

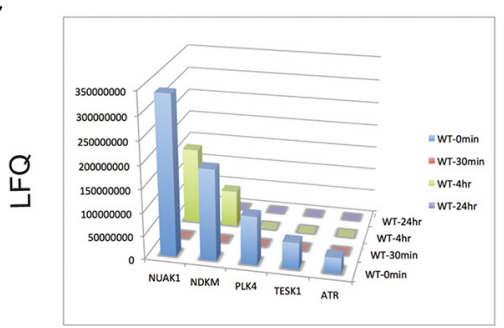

competition binding approaches to assess inhibitor selectivity/potency have identified crizotinib as a potent inhibitor of FAK1 ([22] and HMS Library of Integrated Network-based Cellular Signatures PF02341066 KINOMEscan dataset 20033). Multiple lines of evidence from our work suggest that FAK1 is indeed the relevant target of crizotinib. First, our data confirm that crizotinib treatment inhibits the activation of FAK1, as determined by phosphorylation of tyrosine 397 which correlates with activation of this kinase $[25,26]$. Second, treatment of NF2-null Schwann cells with small molecules inhibitors of FAK 1 result in inhibition of cell proliferation in a dosedependent manner, mimicking the outcomes of crizotinib treatment. Third, and most important, direct inhibition of FAK1 in two NF2-null Schwann cell lines, using siRNA or shRNA, recapitulates the phenotypes observed with crizotinib treatment and confirms that loss of FAK1 is incompatible with proliferation of these cells. Finally, the ability of crizotinib resistant FAK1 mutants to shift and increase the $\mathrm{EC}_{50}$ of crizotinib against SC4 cells suggests that the effects of crizotinib in these cells are mediated largely through FAK1 inhibition. While these studies do not exclude the possible contribution of other targets,
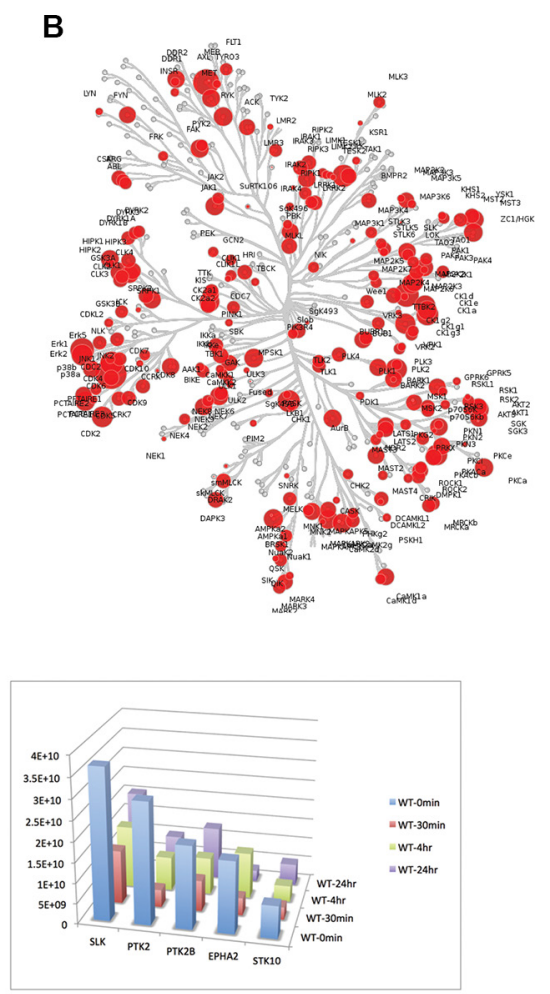

Figure 3: Expression and activity based profiling of the kinome in NF2-null schwann cells. (A) Untreated SC4 cells were extracted for RNA and analyzed by sequencing. The expressed kinases ( $>1$ RPKM) are indicated in yellow on the dendogram, with the size of each circle representing the $\log _{2}$ transformed RPKM value. (B) Untreated SC4 cells were extracted for protein and the activity-based profile of the kinome was assessed by binding to a multiplexed inhibitor bead matrix, followed by elution of bound proteins and identification/ quantification by label-free mass spectrometry. The levels of each kinase identified are indicated in red on the dendogram, with the size of each circle representing the $\log _{2}$ transformed quantification value. (C) Time-dependent activity-based profiles for kinases that met the selection criteria. SC4 cells were treated with $10 \mu \mathrm{M}$ crizotinib and harvested at $0,30^{\prime}, 4 \mathrm{~h}$ and $24 \mathrm{~h}$ post treatment. LFQ $=$ Label-Free quantification values. 
the extent and concordance of the phenotypes observed suggest that inhibition of FAK1 is the major mechanism through which the effects of crizotinib are mediated.

FAK1 (coded by the PTK2 gene) is a cytoplasmic tyrosine kinase shown to be upregulated and activated in a range of solid tumor types. FAK1 promotes cell proliferation, survival, motility and other functions, through kinase-dependent and independent mechanisms [27]. Recent studies in a panel of mesothelioma cells lines revealed that sensitivity to FAK1 inhibition was inverselycorrelated to levels of Merlin expression. The proposed mechanism suggests Merlin loss leads to destabilization of cell:cell junctions, which causes the cells to become more reliant on cell-ECM interactions for survival and proliferation. As FAK1 is a critical effector of these proliferative/survival signals, the consequences of FAK1 inhibition in the context of NF2 loss are detrimental to the cells [18]. Other studies examining the relationship between Merlin and FAK1 in ovarian cancer cell identified a correlation between Merlin expression levels and sensitivity to FAK1 inhibitors, when cells were grown in an anchorage-independent manner. Intriguingly, stable knockdown of Merlin in resistant cells did not sensitize cells to FAK1 inhibition. This suggests that at least in ovarian cancer cell lines Merlin expression might predict responsiveness to FAK1 inhibitors, although a causal link between Merlin and FAK1 was not identified [17]. These recent findings, along with the data presented in our report,
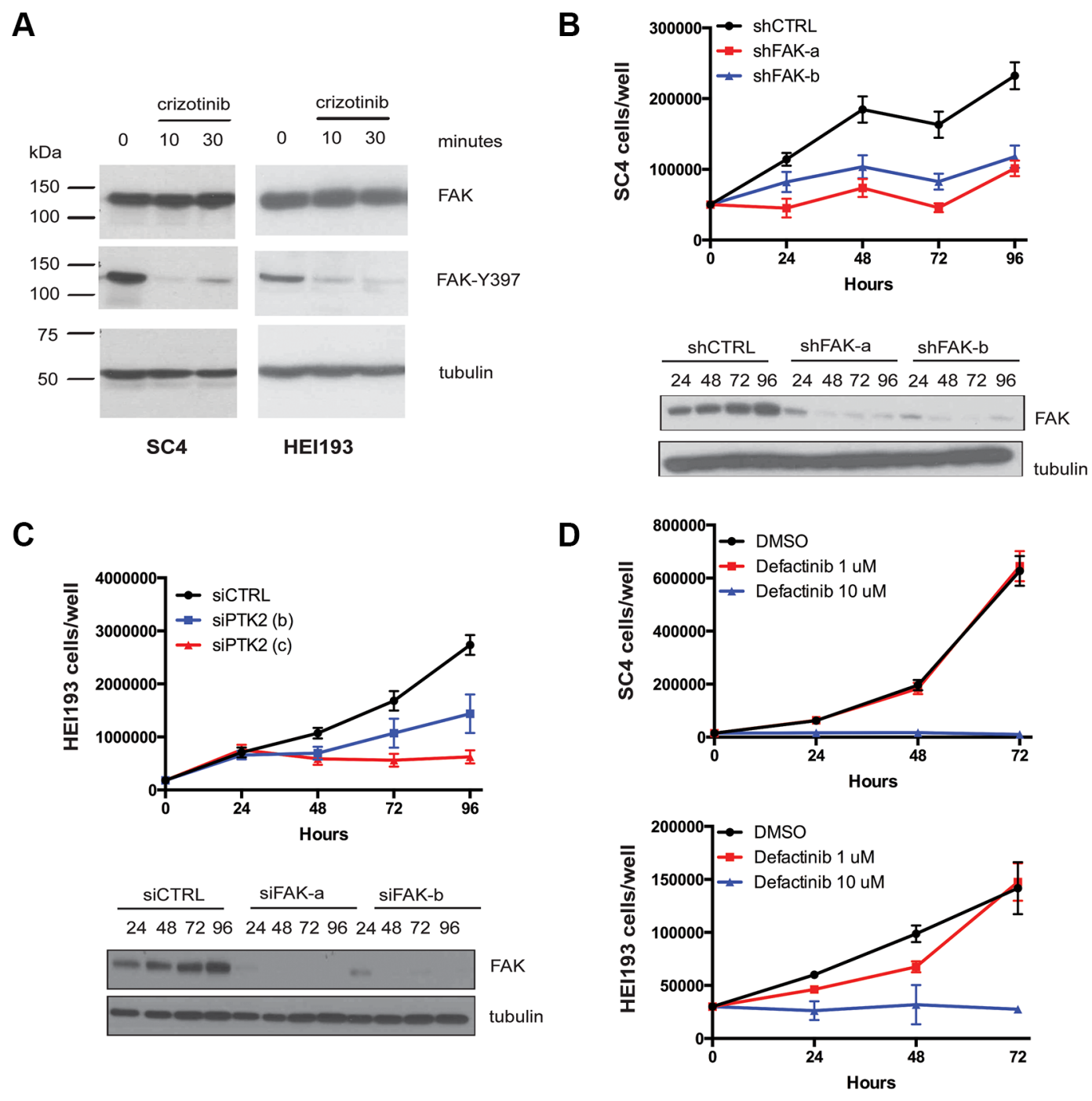

Figure 4: FAK1 is inhibited by crizotinib and required for proliferation of NF2-null schwann cells. (A) Western blot analysis of total FAK1 and p-FAKY ${ }^{397}$ in protein extracts prepared from SC4 and HEI193 cells treated with crizotinib (10 $\mu \mathrm{M}$ for 10' and 30'). Vinculin was used as a loading control. (B) Cell number counts of SC4 cells and western blot analysis of total FAK1 expression in cells treated with 2 independent shRNAs against FAK1 (shFAK-a, shFAK-b) or scrambled control (shCTRL). (C) Cell number counts of HEI193 cells and western blot analysis of total FAK1 expression in cells treated with 2 independent shRNAs against FAK1 (siFAK-a, siFAK-b) or scrambled control (siCTRL). (D) SC4 or HEI193 cells treated with defactinib at the indicated doses or with DMSO control, daily for 3 days. In all counting experiments cell numbers were scored daily and each time point was done in triplicate. The data shown represent the mean of 3 independent experiments. Error bars $=$ SD. 
strongly implicate FAK1 as a critical effector and as a relevant target in the context of Merlin deficiency.

From a clinical standpoint, crizotinib represents an excellent candidate for evaluation in NF2. It is well tolerated and has already received FDA approval for the treatment of patients with metastatic non-small cell lung cancer that are positive for activating ALK fusions. In addition, crizotinib is being evaluated in phase I/II trials in children with relapsed/ refractory solid tumors and primary CNS tumors such as neuroblastoma (NCT00939770). In addition to assessing the efficacy of crizotinib against NF2-deficient schwannomas, the activity of crizotinib against other NF2-null tumors, such as meningiomas and mesotheliomas, should be evaluated. While crizotinib was developed as an MET/ALK inhibitor, there are a number of inhibitors specifically developed against FAK1 that are currently in various stages of clinical trial. These include GSK2256098, PND-1186 (VS-4718),
PF-271 (VS-6062) and defactinib (VS-6063) [27]. Once approved, these drugs could represent additional therapeutic options for NF2-null tumors and should thus be evaluated. Interestingly, our studies with PND-1186 and defactinib suggest a similar potency of these drugs compared to crizotinib. This suggests that the inhibitory capacity of crizotinib against FAK1 is sufficient to elicit a favorable effect. However, a true comparison of these drugs will ultimately require assessment in a clinical trial setting.

In summary, our studies identify an FDA approved drug, crizotinib, which shows in vitro and in vivo efficacy against NF2-null schwannomas. Furthermore, our studies identify the mechanism of action, through the inhibition of FAK1. Given the lack of curative options for NF2 and the favorable tolerability of this drug, crizotinib represents an excellent candidate for clinical testing in patients harboring NF2-deficient tumors.
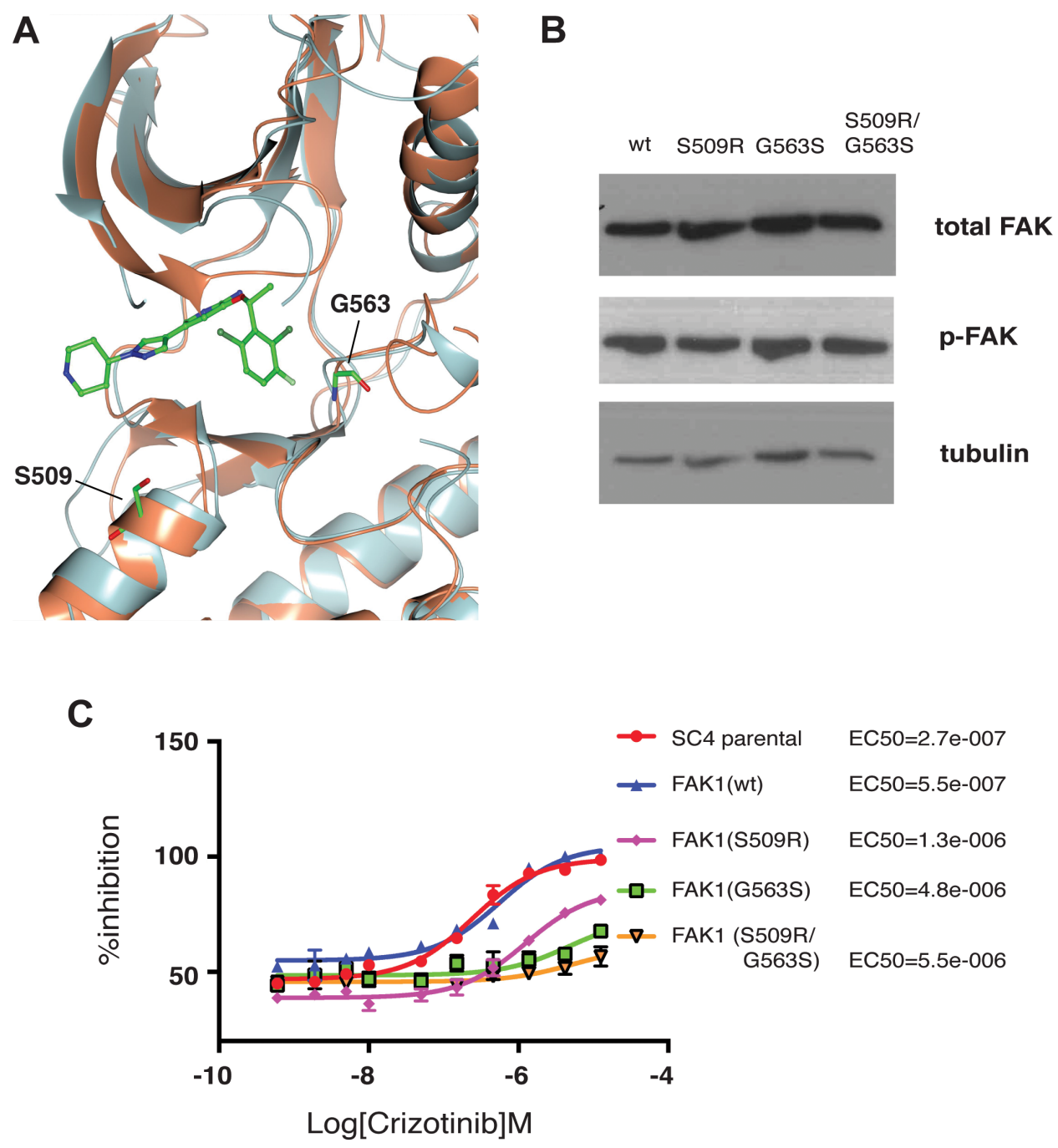

Figure 5: Crizotinib-resistant FAK1 mutants rescue proliferation of treated NF2-null schwann cells. (A) Superimposition of the FAK1 (orange) and ALK (gray) kinase domains with crizotinib (ball and stick). Residues Glycine 509 (G509) and Serine 563 (S563) are highlighted (ball and stick). (B) Western blot analysis of the different FAK1 mutant expression in stably transfected SC4 cells. Vinculin was used as a loading control (C) 10-point dose response curves assessing EC50 of crizotinib in SC4 cells stably expressing crizotinib resistant FAK1 mutants. Calculated EC50 for each clone is indicated to the right. The data shown represents the mean of 3 independent experiments, each done in quadruplicate. Error bars $=\mathrm{SD}$. 


\section{MATERIALS AND METHODS}

\section{Cell culture conditions}

The SC4 and HEI193 cell lines were obtained from The House Ear Institute and ATCC, respectively. The hSC $2 \lambda$ cells are a gift from Dr. Margaret Wallace (U. of Florida) [28]. All cell lines were authenticated by short tandem repeat (STR) DNA profiling (DDC Medical) (March 2015) and verified to be clean of mycoplasma contamination using a PCR based approach. Cells were grown in low-glucose DME, $10 \%$ fetal calf serum, $1 \times$ non-essential amino acids and $100 \mathrm{IU} / \mathrm{ml}$ penicillin-streptomycin. Transfections were performed with Lipofectamine 2000 (Invitrogen), following manufacturer's instructions.

\section{Western blot analysis}

Protein extracts were prepared with RIPA lysis buffer $(50 \mathrm{mM}$ TRIS-HCl, pH 7.5, 1\% Nonidet P-40, $0.25 \%$ sodium deoxycholate, $150 \mathrm{mM} \mathrm{NaCl}, 1 \mathrm{mM}$ EGTA, $1 \mathrm{mM}$ sodium orthovanadate, and $1 \mathrm{mM} \mathrm{NaF}$ ). The following antibodies were used according to manufactures' instructions: MET, FAK1 and FAK-pY397 (cat\# 3127, 3285, 3283 - Cell Signaling Technology).

\section{RNAi-mediated knockdown of FAK}

HEI193 and SC4 cells were transfected using Lipofectamine 2000 (Invitrogen, NY, USA), according to the manufacturer's instructions. Specifically HEI193 cells were transfected with siRNA duplexes targeting FAK1 [ID\# SR303877A- GCAAUGGAGCCGAGUAUUA AAGGUCT and SR303877B- AGAAGAUACUUACA CCAUGCCCUCA] as well as a non-targeting siRNA control[ID\#SR30004-CGUUAAUCGCGUAUAAUACG CGUAT] (Origene, Rockville, USA) at a concentration of $30 \mathrm{nM}$. SC4 cells were transfected with TRC mouse shRNA individual clones targeting FAK1 [ID \# TRCN0000 $023484-5$ '-CGGTCCAATGACAAGGTATAT-3' and TRCN0000023486-5'-CCAACCTTAATAGAGAAGAA A-3'] (GE Dharmacon, Lafayette, USA).

\section{CRISPR/Cas9 editing of MET}

Guide sequences from exon 1 of the MET locus (g\#1- GTTTACTGACATACGCGGCT and g\#2GTTCATCTCAGACTTCACTA) were cloned into the pX459 vector (Addgene). SC4 cells were transfected with $8 \mu \mathrm{g}$ of plasmid into the SC4 cells using Lipofectamine 2000, according to manufacturer's instructions. Cells were selected for 48 hours in puromycin and individual clones were picked and expanded after 7 days. Clones were analyzed by western blotting to determine loss of MET expression.

\section{Cell proliferation assays}

30,000 cells/well were plated in 12-well dishes in triplicate. At indicated time points, cells from individual wells were trypsinized and counted using a Coulter counter (Z1 series, Beckman Coulter). Cell growth media was replaced daily. For measurement of cell proliferation, cells were plated at the numbers indicated in the figures and BrdU Proliferation Assay (Millipore) was used according to the manufacturer's instructions. Statistical significance was determined by a two-tailed student's $t$-test. Each condition at each time point represents the mean of 3 experiments in triplicate for a total of 9 wells.

\section{Generation of FAK1 mutants and stable SC4 clones}

The FAK1 mutant constructs were created by PCR amplification of FAK1 cDNA from the pLV-Neo-CD2FAK1 (addgene \#37013) and cloning into pcDNA3.1 using NheI and NotI sites (primer sequences Fak-Nhe1F 5'-aaaagctagcaaaATGGCAGCTGCTTACCTTGACC-3', Fak-Not1R 5'-aaaagcggccgcaaaTCAGTGTGGTCTCG TCTGCC-3'). Site directed mutagenesis was performed using the Agilent Quick Change SiteDirected Mutagenesis Kit (cat\# 200518-5). Primers were designed using the SDM design tool on the Agilent website. Primer sequences: FAKS509R: $5^{\prime}$-gcacacttggagagctgaggagatttttgcaagtaaggaaat- $3^{\prime}$ and $5^{\prime}$-attccttacttgcaaaaatctcctcagctctccaagtgtgc- $3^{\prime}$. FAKG563S: $5^{\prime}$-ctggtgtcctcaaatgattgtgtaaaattaagcgactttggattatccc- $3^{\prime}$ and $5^{\prime}$ - gggataatccaaagtcgettaattttacacaatcatttgaggacaccag-3'. Bacterial clones were isolated and sequence verified. SC4 Cells were transfected with $8 \mu \mathrm{g}$ of plasmid DNA, using Lipofectamine 2000 (cat\# 11668019), and were placed under G418 selection, $150 \mathrm{ug} / \mathrm{ml}$ until individual clones could be picked and expanded for experimentation.

\section{RNA sequencing data}

RNA from SC4 cells was extracted using TRIZOL reagent. $5-10 \mu \mathrm{g}$ of total RNA was used to isolate poly(A) RNA using the micropoly(A) purist kit (Ambion). The whole transcriptome library kit (Life Technologies) was used to prepare paired-end sequencing libraries. Briefly, $100 \mathrm{ng}$ of poly(A) RNA was enzymatically fragmented to an average size of $150 \mathrm{nt}$, ligated to directional adapters and reverse-transcribed, and the cDNA was size-selected on denaturing $6 \%$ TBE-Urea polyacrylamide gels, amplified for 15 cycles with barcoded primers and purified using AmpPure XP beads. The resulting library was quantified using Bioanalyzer (Agilent) and Taqman (Life Technologies) assays. The barcoded library combined was used at a final concentration of $0.4 \mathrm{pM}$ in emulsion PCR to link to the sequencing beads. For analysis, the sequencing reads in color-space were mapped to the $\mathrm{mm} 9$ 
genome using Tophat [29]. The number of reads falling into each gene defined in the RefSeq gene annotations was quantified using HTSeq-count [30]. Samples from three independent experiments were sequenced, combined, and analyzed to produce the final DESeq data. The RNA-Seq data is publicly available through the NCBI GEO database with accession number GSE61528.

\section{Determination of $\mathrm{EC}_{50} \mathrm{~s}$ and dose response analysis}

To determine the $\mathrm{EC}_{50}$ of crizotinib 2500 cells from each clone were plated, in quadruplicate, in a solid white cell culture 384-well plate. Crizotinib was added to the wells in a three-fold ten-point dilution series ranging from $12.5 \mu \mathrm{M}$ to $0.0006 \mu \mathrm{M}$. Crizotinib activity was evaluated 24 hours after treatment using CellTiter-Glo (Promega, USA) as a read-out of viability. The $\mathrm{EC}_{50}$ values were calculated using Graphpad Prism.

\section{In vivo tumor models and imaging}

All animal experiments were approved by the Scripps Institutional Animal Care and Use Committee and performed in accordance with relevant institutional and national guidelines. The use of the orthotopic tumor

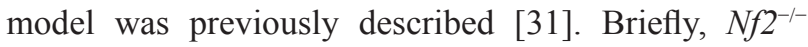
SC4 Schwann cells were transduced by lentiviruses carrying pLuc-mCherry and sorted by FACS. $5 \times 10^{4}$ cells were transplanted into the sciatic nerve sheath of NOD/SCID mice ( 8 weeks of age) by intraneural injection. Tumor progression was monitored every 3 days by bioluminescence imaging (BLI) according to the manufacturer's instructions on an IVIS-200 system (Xenogen, San Francisco, CA).

\section{MIB chromatography}

Cells were treated with $10 \mu \mathrm{M}$ crizotinib for the indicated time points and tumors were harvested as previously described [16]. Protein lysates containing $3 \mathrm{mg}$ of total protein each were brought to $1 \mathrm{M} \mathrm{NaCl}$ and passed through columns of inhibitor-conjugated beads (Bisindoylmaleimide-X,SB203580, Dasatinib, PP58, VI16832, and CTx-0294885) to isolate protein kinases. Kinase-bound inhibitor beads were washed with high- and low-salt buffers and $0.1 \%$ SDS before elution by boiling in $0.5 \%$ SDS with $1 \% \beta$-mercaptoethanol in $0.1 \mathrm{M}$ Tris- $\mathrm{HCl}$ ( $\mathrm{pH}$ 6.8). Proteins were purified using chloroform $/$ methanol extraction and resuspended in $50 \mathrm{mM}$ HEPES ( $\mathrm{pH} 8)$. Samples were digested overnight at $37^{\circ} \mathrm{C}$ using sequencing grade modified trypsin (Promega). Peptides were fractionated with Mini SCX spin columns and cleaned with PepClean C18 Spin Columns (Thermo Scientific) before MS analysis.

\section{MS analysis}

MS and MS/MS data were acquired with a Q Exactive ${ }^{\mathrm{TM}}$ Hybrid Quadrupole-Orbitrap Mass SpectroMETer (Thermo) equipped with an EASYnLC1000 nano UHPLC (Thermo). Each individual sample was resuspended in $20 \mu \mathrm{L}$ of sample buffer $(2 \%$ ACN , $0.1 \%$ TFA ) and injected ( $5 \mu \mathrm{L})$ onto the Thermo Scientific EASY-Spray column $(\mathrm{C} 18,2 \mu \mathrm{M}, 100 \mathrm{~A}$, $75 \mu \mathrm{M}$ X $25 \mathrm{~cm})$. A linear gradient from $5 \% \mathrm{~B}\left(\mathrm{CH}_{3} \mathrm{CN}\right.$ $+0.1 \%$ formic acid) to $25 \% \mathrm{~B}$ in 150 minutes and then $25 \%$ B to $45 \%$ B in 15 minutes, followed by $45 \%$ B to $95 \% \mathrm{~B}$ in 5 minutes and pumping $95 \%$ of $\mathrm{B}$ for additional 10 minutes. MS and MS/MS data were analyzed by MaxQuant 1.5.0.0 with a UniProtKB database using label free quantifications.

\section{Statistical analysis}

To assess the anti-tumor activity of crizotinib in vivo, the natural log-transformed flux readings were used to reflect tumor growth. The trends of tumor growth over time (followed up by days) were examined between control and treatment groups using a mixedeffect model with the random effect at the mouse level. A linear function, in days, was determined to provide the best fit without random slope on followup days. A likelihood ratio testing nested models (with versus without the interaction term of group and time) was used to examine if trends are significantly different overall between groups. The tumor weights were first examined by a Shapiro-Wilk test to assess data normality and a Variance ratio test was used to examine the equality of variances between two groups. The differences between the control and experimental cohorts were analyzed by a $t$-test with equal variances. Statistical analyses were performed using Stata 12.0 (StataCorp LP, Texas, USA).

\section{ACKNOWLEDGMENTS}

We thank the Scripps Genomics Core for their assistance with RNA-Seq experiments and Gautam Shankar from the Scripps Informatics Core. We thank members of the Kissil lab for critical reading of the manuscript.

This work was supported in part by the NIH (NS077952 and CA124495 to JLK and GM101141 to GLJ) and the Children's Tumor Foundation (JLK). SM is a recipient of a Young Investigator Award from the Children's Tumor Foundation.

\section{CONFLICTS OF INTEREST}

The authors declare no conflicts of interests. 


\section{Authors' contributions}

S.T., S.M., and J.L.K. conceived and designed the study, designed experiments, interpreted results and wrote the manuscript. S.K., Q.L. and G.L.J. designed experiments, assisted in interpretation and edited the manuscript.

\section{REFERENCES}

1. Gusella JF, Ramesh V, MacCollin M, Jacoby LB. Merlin: the neurofibromatosis 2 tumor suppressor. Biochim Biophys Acta. 1999; 1423:M29-36.

2. Stamenkovic I, Yu Q. Merlin, a "magic" linker between extracellular cues and intracellular signaling pathways that regulate cell motility, proliferation, and survival. Curr Protein Pept Sci. 2010; 11:471-84.

3. Morrison H, Sherman LS, Legg J, Banine F, Isacke C, Haipek CA, Gutmann DH, Ponta H, Herrlich P. The NF2 tumor suppressor gene product, merlin, mediates contact inhibition of growth through interactions with CD44. Genes Dev. 2001; 15:968-80.

4. Torres-Martin M, Lassaletta L, San-Roman-Montero J, De Campos JM, Isla A, Gavilan J, Melendez B, Pinto GR, Burbano RR, Castresana JS, Rey JA. Microarray analysis of gene expression in vestibular schwannomas reveals SPP1/MET signaling pathway and androgen receptor deregulation. Int J Oncol. 2013.

5. Kissil JL, Wilker EW, Johnson KC, Eckman MS, Yaffe MB, Jacks T. Merlin, the product of the Nf2 tumor suppressor gene, is an inhibitor of the p21-activated kinase, Pak1. Mol Cell. 2003; 12:841-9.

6. Yi C, Wilker EW, Yaffe MB, Stemmer-Rachamimov A, Kissil JL. Validation of the p21-activated kinases as targets for inhibition in neurofibromatosis type 2. Cancer Res. 2008; 68:7932-7.

7. Xiao GH, Gallagher R, Shetler J, Skele K, Altomare DA, Pestell RG, Jhanwar S, Testa JR. The NF2 tumor suppressor gene product, merlin, inhibits cell proliferation and cell cycle progression by repressing cyclin D1 expression. Mol Cell Biol. 2005; 25:2384-94.

8. Yi C, Troutman S, Fera D, Stemmer-Rachamimov A, Avila JL, Christian N, Persson NL, Shimono A, Speicher DW, Marmorstein R, Holmgren L, Kissil JL. A tight junction-associated Merlin-angiomotin complex mediates Merlin's regulation of mitogenic signaling and tumor suppressive functions. Cancer Cell. 2011; 19:527-40.

9. Shrestha Y, Schafer EJ, Boehm JS, Thomas SR, He F, Du J, Wang S, Barretina J, Weir BA, Zhao JJ, Polyak K, Golub TR, Beroukhim R, et al. PAK1 is a breast cancer oncogene that coordinately activates MAPK and MET signaling. Oncogene. 2011.

10. Chow HY, Stepanova D, Koch J, Chernoff J. p21-Activated kinases are required for transformation in a cell-based model of neurofibromatosis type 2. PLoS One. 2010; 5:e13791.
11. Cui JJ, Tran-Dube M, Shen H, Nambu M, Kung PP, Pairish M, Jia L, Meng J, Funk L, Botrous I, McTigue M, Grodsky N, Ryan K, et al. Structure based drug design of crizotinib (PF-02341066), a potent and selective dual inhibitor of mesenchymal-epithelial transition factor (c-MET) kinase and anaplastic lymphoma kinase (ALK). J Med Chem. 2011; 54:6342-63.

12. Saydam O, Ozdener GB, Senol O, Mizrak A, Prabhakar S, Stemmer-Rachamimov AO, Breakefield XO, Brenner GJ. A novel imaging-compatible sciatic nerve schwannoma model. J Neurosci Methods. 2011; 195:75-7.

13. Wong HK, Lahdenranta J, Kamoun WS, Chan AW, McClatchey AI, Plotkin SR, Jain RK, di Tomaso E. Antivascular endothelial growth factor therapies as a novel therapeutic approach to treating neurofibromatosis-related tumors. Cancer Res. 2010; 70:3483-93.

14. Liu X, Wang Q, Yang G, Marando C, Koblish HK, Hall LM, Fridman JS, Behshad E, Wynn R, Li Y, Boer J, Diamond S, $\mathrm{He}$ C, et al. A novel kinase inhibitor, INCB28060, blocks c-MET-dependent signaling, neoplastic activities, and cross-talk with EGFR and HER-3. Clin Cancer Res. 2011; 17:7127-38.

15. Stuhlmiller TJ, Earp HS, Johnson GL. Adaptive reprogramming of the breast cancer kinome. Clin Pharmacol Ther. 2014; 95:413-5.

16. Duncan JS, Whittle MC, Nakamura K, Abell AN, Midland AA, Zawistowski JS, Johnson NL, Granger DA, Jordan NV, Darr DB, Usary J, Kuan PF, Smalley DM, et al. Dynamic reprogramming of the kinome in response to targeted MEK inhibition in triple-negative breast cancer. Cell. 2012; 149:307-21.

17. Shah NR, Tancioni I, Ward KK, Lawson C, Chen XL, Jean C, Sulzmaier FJ, Uryu S, Miller NL, Connolly DC, Schlaepfer DD. Analyses of merlin/NF2 connection to FAK inhibitor responsiveness in serous ovarian cancer. Gynecol Oncol. 2014; 134:104-11.

18. Shapiro IM, Kolev VN, Vidal CM, Kadariya Y, Ring JE, Wright Q, Weaver DT, Menges C, Padval M, McClatchey AI, Xu Q, Testa JR, Pachter JA. Merlin deficiency predicts FAK inhibitor sensitivity: a synthetic lethal relationship. Sci Transl Med. 2014; 6:237ra68.

19. Zhang S, Wang F, Keats J, Zhu X, Ning Y, Wardwell SD, Moran L, Mohemmad QK, Anjum R, Wang Y, Narasimhan NI, Dalgarno D, Shakespeare WC, et al. Crizotinib-resistant mutants of EML4-ALK identified through an accelerated mutagenesis screen. Chem Biol Drug Des. 2011; 78:999-1005.

20. Katayama R, Shaw AT, Khan TM, Mino-Kenudson M, Solomon BJ, Halmos B, Jessop NA, Wain JC, Yeo AT, Benes C, Drew L, Saeh JC, Crosby K, et al. Mechanisms of acquired crizotinib resistance in ALK-rearranged lung Cancers. Sci Transl Med. 2012; 4:120ra17.

21. Zou HY, Li Q, Lee JH, Arango ME, McDonnell SR, Yamazaki S, Koudriakova TB, Alton G, Cui JJ, Kung PP, Nambu MD, Los G, Bender SL, et al. An orally available small-molecule inhibitor of c-Met, PF-2341066, exhibits 
cytoreductive antitumor efficacy through antiproliferative and antiangiogenic mechanisms. Cancer Res. 2007; 67:4408-17.

22. Davis MI, Hunt JP, Herrgard S, Ciceri P, Wodicka LM, Pallares G, Hocker M, Treiber DK, Zarrinkar PP. Comprehensive analysis of kinase inhibitor selectivity. Nat Biotechnol. 2011; 29:1046-51.

23. Shaw AT, Ou SH, Bang YJ, Camidge DR, Solomon BJ, Salgia R, Riely GJ, Varella-Garcia M, Shapiro GI, Costa DB, Doebele RC, Le LP, Zheng Z, et al. Crizotinib in ROS1-rearranged non-small-cell lung cancer. N Engl J Med. 2014; 371:1963-71.

24. Huber KV, Salah E, Radic B, Gridling M, Elkins JM, Stukalov A, Jemth AS, Gokturk C, Sanjiv K, Stromberg K, Pham T, Berglund UW, Colinge J, et al. Stereospecific targeting of MTH1 by (S)-crizotinib as an anticancer strategy. Nature. 2014; 508:222-7.

25. Schaller MD, Hildebrand JD, Shannon JD, Fox JW, Vines RR, Parsons JT. Autophosphorylation of the focal adhesion kinase, pp125FAK, directs SH2-dependent binding of pp60src. Mol Cell Biol. 1994; 14:1680-8.

26. Calalb MB, Polte TR, Hanks SK. Tyrosine phosphorylation of focal adhesion kinase at sites in the catalytic domain regulates kinase activity: a role for Src family kinases. Mol Cell Biol. 1995; 15:954-63.
27. Sulzmaier FJ, Jean C, Schlaepfer DD. FAK in cancer: mechanistic findings and clinical applications. Nat Rev Cancer. 2014; 14:598-610.

28. Rahrmann EP, Moriarity BS, Otto GM, Watson AL, Choi K, Collins MH, Wallace M, Webber BR, Forster CL, Rizzardi AE, Schmechel SC, Ratner N, Largaespada DA. Trp53 haploinsufficiency modifies EGFR-driven peripheral nerve sheath tumorigenesis. Am J Pathol. 2014; 184:2082-98.

29. Trapnell C, Pachter L, Salzberg SL. TopHat: discovering splice junctions with RNA-Seq. Bioinformatics. 2009; 25:1105-11.

30. Anders S, Huber W. Differential expression analysis for sequence count data. Genome Biol. 2010; 11:R106.

31. Licciulli S, Maksimoska J, Zhou C, Troutman S, Kota S, Liu Q, Duron S, Campbell D, Chernoff J, Field J, Marmorstein R, Kissil JL. FRAX597, a small molecule inhibitor of the p21-activated kinases, inhibits tumorigenesis of neurofibromatosis type 2 (NF2)-associated Schwannomas. J Biol Chem. 2013; 288:29105-14. 